\title{
Properties of curvilinear vection
}

\author{
XAVIER M. SAUVAN \\ University Hospital, Zurich, Switzerland \\ and \\ CLAUDE BONNET \\ Université René Descartes (Paris V), Paris, France
}

\begin{abstract}
Approximately linear relationships were observed between contrast, spatial frequency, temporal frequency, or velocity of stimulation and perceived velocity of curvilinear vection-that is, a visually induced self-motion in a curved path. Similarly, linear relationships were also found between the perceived degree of curvature of curvilinear vection and spatial frequency or velocity of stimulation. Since the perceived velocity of curvilinear vection varies with contrast, spatial frequency, temporal frequency, and angular velocity, and the perceived degree of curvature of curvilinear vection varies only with spatial frequency and angular velocity, peripheral vision is not sufficient for computing accurately the curvilinear component of induced self-motion in a curved path. Concurrently, it was shown that the perceived direction of curvilinear vection is not always unambiguously perceived (Sauvan \& Bonnet, 1989). Consequently, it is suggested that two different types of visual processing, which involve the peripheral or the central vision, underlie the processing of curvilinear vection.
\end{abstract}

This experimental work is concerned with the study of the properties of curvilinear vection. It is the second part of a more general work, the first part of which is the study of the thresholds of linear vections (Sauvan \& Bonnet, 1988, 1989).

Vections are induced sensations of self-motion. It is possible to induce vections by stimulating only a part of the visual field, for example, the central visual field (see Andersen \& Braunstein, 1985, for rectilinear vection, and Post, 1988, for circular vection). The perceived direction of vection is always opposite to the direction of the stimulation (Berthoz \& Droulez, 1982; Dichgans \& Brandt, 1978). Curvilinear vection corresponds to the sensation of taking a bend, and it contains two components, a translational one and a rotational one (Andersen, 1986). It is similar to the actual observer movement in a curved path, such as moving within a turning vehicle. In the present experimental work, a visual stimulation has been used to induce curvilinear vection. Vections can also be induced, for example, by visual and vestibular stimulations in interaction (Dichgans \& Brandt, 1978). Indeed, the visual and vestibular inputs converge in the vestibu-

The experiments described in this article were carried out in the Laboratory of Experimental Psychology (UA 316 CNRS \& Université René Descartes). We thank C. Tyler for helpful comments on earlier versions of the manuscript and V. Henn and J. Droulez for their advice. We are grateful to $N$. Bonora and $C$. Bogey for their technical assistance and to G. Loakes for improving our English. X.M.S. particularly thanks J. Rauschecker for his helpful comments and his kindness during his stay in the Unit on Neuroethology of the NIMH/NIHAC at Poolesville, MD, USA. Correspondence concerning this article should be addressed to X. Sauvan, Department of Neurology, University Hospital, Frauenklinikstrasse 26, CH-8091 Zurich, Switzerland. lar nuclei (Waespe \& Henn, 1977, 1978) by a route involving the nucleus of the optic tract and the nucleus reticularis tegmenti pontis with respect to the visual inputs (Howard, 1986), at thalamic level (Büttner \& Henn, 1976; Büttner \& Lang, 1979) and at cortical level (Büttner \& Buettner, 1978; Büttner \& Lang, 1979; see also Orban, 1984). Finally, a dominance of the visual system can be observed in the case of circular vection (Büttner \& Henn, 1981; Probst, Straube, \& Bles, 1985), which corresponds to an induced rotation about a vertical axis, or in the case of rectilinear vection (Berthoz \& Droulez, 1982; Berthoz, Pavard, \& Young, 1975), which corresponds to an induced translation in a straight line. This result should be related to the existence of neurons in parietal visual area MST in monkey that strongly respond to optic flow stimuli (Wurtz \& Duffy, 1992).

Experimental studies of locomotion or driving a vehicle in a curved path are usually concerned with real curvilinear movement; they are often carried out within the framework of the computational analysis of optic flow (e.g., Longuet-Higgins \& Prazdny, 1980; Prazdny, 1981, 1983; Rieger, 1983; see also Andersen, 1986). The aim of the present work, therefore, is to study with a psychophysical approach the properties of curvilinear vection as a function of contrast, spatial frequency, temporal frequency, or angular velocity of visual stimuli (Experiments 1 and 2) and to estimate the temporal characteristics of curvilinear vection (Experiment 3). Curvilinear vection corresponds to locomotion or driving a vehicle in a curved path and is induced by stimulating each eye with a different contrast, spatial frequency, temporal frequency, or angular velocity (Sauvan \& Bonnet, 1988, 1989). In a more precise way, every factor that introduces enough 
difference between the apparent velocities of two peripheral moving visual patterns, symmetrically placed with respect to the subject's head, induces curvilinear vection (Sauvan \& Bonnet, 1989). Thresholds of perception of linear vections define a spatiotemporal zone of perception of linear vections, with the thresholds of detection of sinewave gratings motion lying outside of that spatiotemporal area (Sauvan \& Bonnet, 1988, 1989). Curvilinear vection is perceived in the present experimental work by means of peripheral stimulation of low spatial frequency but small area. Perceived velocity and degree of curvature of curvilinear vection vary differently. Moreover, it has been shown that the perceived direction of this vection is not always unambiguously reported (Sauvan \& Bonnet, 1989). It is proposed that the lower and the higher level ambient systems, according to Andersen and Braunstein's (1985) theory, should be involved in the perception of curvilinear vection.

\section{GENERAL METHOD}

\section{Subjects}

Seven healthy young adults -5 emmetropic females (M.B., N.B., I.G., E.R., and C.T.) and two myopic males (R.P. and X.S.) between the ages of 22 and $28-$ took part in two or three experiments.

\section{Apparatus}

The subjects were seated with their head on a chinrest and were directed to gaze at a fixation point placed before them. Two Tektronix monitors 604 (P31) were displayed behind two circular apertures. One aperture subtended a visual angle of $23^{\circ}$. The monitors simulated corresponding retinal points. The distance between the subject's eyes and the screens of the monitors was equal to $25 \mathrm{~cm}$. Leibowitz, Rodemer, and Dichgans (1979) have shown that vection is independent of refractive error. Consequently, the small distance between the monitors and the subject's eyes should not have had an effect on the perceived vection. These apertures were made in a half-sphere located in a dark cabin. The mean luminance of the two monitors was $4.15 \mathrm{~cd} / \mathrm{m}^{2}$. The centers of the screens of these monitors were placed at an eccentricity of $60^{\circ}$ on either side of the subjects' sagittal plane. This experimental situation produced separate stimulation of the two eyes (dichoptic vision) such that each eye viewed only the monitor in its temporal field. The stimuli were vertical sine-wave gratings moving in the nasotemporal direction and generated on the screens of the monitors by a "Picasso"' CRT Image Generator under computer control (Z80 microcomputer system). The contrast of the gratings is defined as $\left(L_{\max }-L_{\min }\right) /$ $\left(L_{\max }+L_{\min }\right)$, in which $L_{\max }$ and $L_{\min }$ are the maximum and minimum luminances of the latter, respectively. The subjects and the Image Generator under computer control were located in two different rooms to reduce the noise in the experimental room as much as possible.

\section{Procedure}

The purpose of these experiments was to observe the effect of four variables on the perception of curvilinear self-motion induced by sinusoidal drifting gratings. Variables were contrast $(C)$, spatial frequency $(S F)$, temporal frequency $(T F)$, and angular velocity $(V=T F / S F)$. Five series of asymmetrical visual stimuli were used. The asymmetry $D(X)$ of the stimuli was given by the difference between the value $X_{1}$ of a given variable $X$ on one of the two monitors and the value $X_{2}$ of the same variable on the other monitor $\left[D(X)=X_{1}-X_{2}\right] . D(X)$ was set at a fixed supraliminal value to induce a curvilinear vection (Sauvan \& Bonnet, 1988, 1989). $D(X)$ was equal to $15 \%$ for contrast, $0.20 \mathrm{c} / \mathrm{deg}$ for spatial fre- quency, $3 \mathrm{~Hz}$ for temporal frequency, and about $6 \mathrm{deg} / \mathrm{sec}$ for angular velocity. Such asymmetrical visual stimuli are powerful in inducing curvilinear vection (Sauvan \& Bonnet, 1988, 1989). A single value of one of the four variables quoted above was presented on each trial of the series, with the exception of the fifth series, in which the variable was a combination of the contrast and the velocity, which varied reciprocally so that the higher the angular velocity, the lower the contrast, and vice versa. In the contrast condition, contrast varied between $35.4 \%$ and $56.4 \%\left(C_{1}\right)$ on one monitor and between $20.4 \%$ and $41.4 \%\left(C_{2}\right)$ on the other. In the spatial frequency condition, spatial frequency varied between 0.23 and $0.60 \mathrm{c} / \mathrm{deg}\left(S F_{1}\right)$ on one monitor and between 0.43 and $0.80 \mathrm{c} / \mathrm{deg}$ $\left(S F_{2}\right)$ on the other monitor. In the temporal frequency condition, the temporal frequency varied between 4.6 and $8.14 \mathrm{~Hz}\left(T F_{1}\right)$ on one monitor and between 1.6 and $5.14 \mathrm{~Hz}\left(T F_{2}\right)$ on the other. Finally, in the velocity condition, angular velocity varied between 4.37 and $10.30 \mathrm{deg} / \mathrm{sec}\left(V_{1}\right)$ on one monitor and between 10.30 and $16.02 \mathrm{deg} / \mathrm{sec}\left(V_{2}\right)$ on the other. Angular velocity values were obtained by using a frequency matrix $(T F / S F)$, and they were selected from one diagonal of that matrix. In all conditions, the values on both monitors varied in the same way so that the linear correlation coefficient between those sets of values was equal to 1 . Moreover, when one of the four variables varied, the values of the three other variables, when they were constant, were close on both monitors to those which defined the optimum of perception of linear vections, that is, $10.2 \mathrm{deg} / \mathrm{sec}$ for angular velocity, $0.49 \mathrm{c} / \mathrm{deg}$ for spatial frequency, $5 \mathrm{~Hz}$ for temporal frequency, and $60 \%$ for contrast (Sauvan \& Bonnet, 1989). Hulk and Rempt (1983) also found that angular velocities from 10 to $15 \mathrm{deg} / \mathrm{sec}$ were the most effective in inducing peripheral vertical vection. The difference of velocity between the two sine-wave drifting gratings $\left[D(V)=V_{1}-V_{2}\right]$ was constant from one trial to another. This relative velocity was equal to zero in the contrast condition, $6.25 \mathrm{deg} / \mathrm{sec}$ in the temporal frequency condition, and about $6 \mathrm{deg} / \mathrm{sec}$ in the velocity condition. There was a logical exception in the spatial frequency condition, in which the possible effect of the relative velocity variation was neutralized by using relative velocities ranging from 2.50 to $4.65 \mathrm{deg} / \mathrm{sec}$. In four trials, we used a subliminal difference of velocity, which did not induce curvilinear vection; in the other two trials, we used a near liminal difference, which induced weak curvilinear vection (Sauvan \& Bonnet, 1988, 1989). It is presumed that any variation of curvilinear vection would be related to the relative velocity variation in the spatial frequency condition.

There were either six or seven trials per series. Indeed, a larger number made it difficult for the subjects to compare their perceptions of curvilinear vection within a given series.

The duration of each trial was $30 \mathrm{sec}$. The conditions were presented in a different random order to each subject, and the trials of the series of stimuli were also chosen at random.

The subjects were first trained to the situation before the experiment started. During the training, a series of stimuli was presented to each subject to verify if he or she perceived curvilinear vection at each trial. The subjects reported if they perceived induced selfmotion in a curved path and if they perceived its direction. The perceived direction of induced self-motion in a curved path was the same during each 30 -sec stimulus. On the whole, it has been found that subjects perceive an induced self-motion in a curved path toward the lowest of the two motions, that is, the lowest apparent velocity (Sauvan \& Bonnet, 1989). At the end of each session and during the training, the subjects were asked if they had already experienced induced self-motion when they were seated in an unmoving train and another train was moving in their peripheral visual field. If they answered affirmatively, they were asked whether the induced-self-motion experience in the experiment or during the training was weaker or stronger than their real-life experience. Furthermore, the subjects were asked whether this induced-self-motion experience was weaker or stronger than their real-life experience of moving in a vehicle. 
Finally, "dummy" series of stimuli, in which stimuli did not induce self-motion or induced the same perception of vection, were used during the training, at the end of several arbitrary chosen sessions, to verify the subjects' perception of induced self-motion.

\section{Statistical Tests}

Two statistical programs were used: VAREDI for the analysis of variance in Experiments 1 and 2 and STAT-ITCF for regression analysis in Experiments 1 and 2. VAREDI was developed in the Laboratory of Experimental Psychology.

\section{EXPERIMENT 1}

The aim of the present experiment was to study the variations of the perceived velocity of curvilinear vection as a function of contrast, spatial frequency, temporal frequency, and angular velocity (or contrast and angular velocity varying reciprocally, in order to test the relative roles of angular velocity and contrast).

\section{Method}

The five conditions described in the General Method section were presented to 6 trained subjects (M.B., N.B., R.P., E.R., C.T., and X.S.). A method of direct estimation was used (Bonnet, 1986) in which the subjects' task was to estimate the perceived velocity of curvilinear vection with an 11-point scale. The subjects were told that a rating of 0 should be given if they did not perceive any vection, up to a rating of 10 to be given to the strongest perception of curvilinear vection with respect to its velocity. During their training, the subjects perceived all of the degrees of curvilinear vection that they encountered later during the experiment so that they would be able to estimate the strongest perception of curvilinear vection in all of the conditions.

\section{Results and Discussion}

The independent variables (contrast, spatial frequency, temporal frequency, or angular velocity) varied in the same way on both monitors (see General Method section). Abscissa values represent stimuli for only one eye in order to make figures intelligible. Perceived velocity of curvilinear vection varied with contrast $[F(6,30)=5.73, p<$ $.01]$, and this variation was linear $[F(1,5)=6.89, p<$ $.05 ; r=.76]$. The equation of the linear function between contrast $(C)$ and perceived velocity of curvilinear vection $(V V)$ was $V V= \pm 139.03 \cdot C-21.89$ (Figure 1A).

Perceived velocity of curvilinear vection varied with spatial frequency $[F(5,25)=26.22, p<.01]$, and this variation was linear $[F(1,4)=73.4, p<.01 ; r=$ $-.974]$. The equation of the linear function between spatial frequency $(S F)$ and perceived velocity of curvilinear vection was $V V=-149.88 \cdot S F+102.87$ (Figure 1B).

Perceived velocity of curvilinear vection varied with temporal frequency $[F(6,30)=6, p<.01]$, and this variation was linear $[F(1,5)=88.78, p<.01 ; r=.973]$. The equation of the linear function between temporal frequency $(T F)$ and perceived velocity of curvilinear vection was $V V=11.98 \cdot T F-30.58$ (Figure $2 \mathrm{~A}$ ).

Perceived velocity of curvilinear vection varied with angular velocity $[F(6,30)=18.81, p<.01]$, and this variation was linear $[F(1,5)=4.71, p<.01 ; r=.95]$. The equation of the linear function between angular ve-
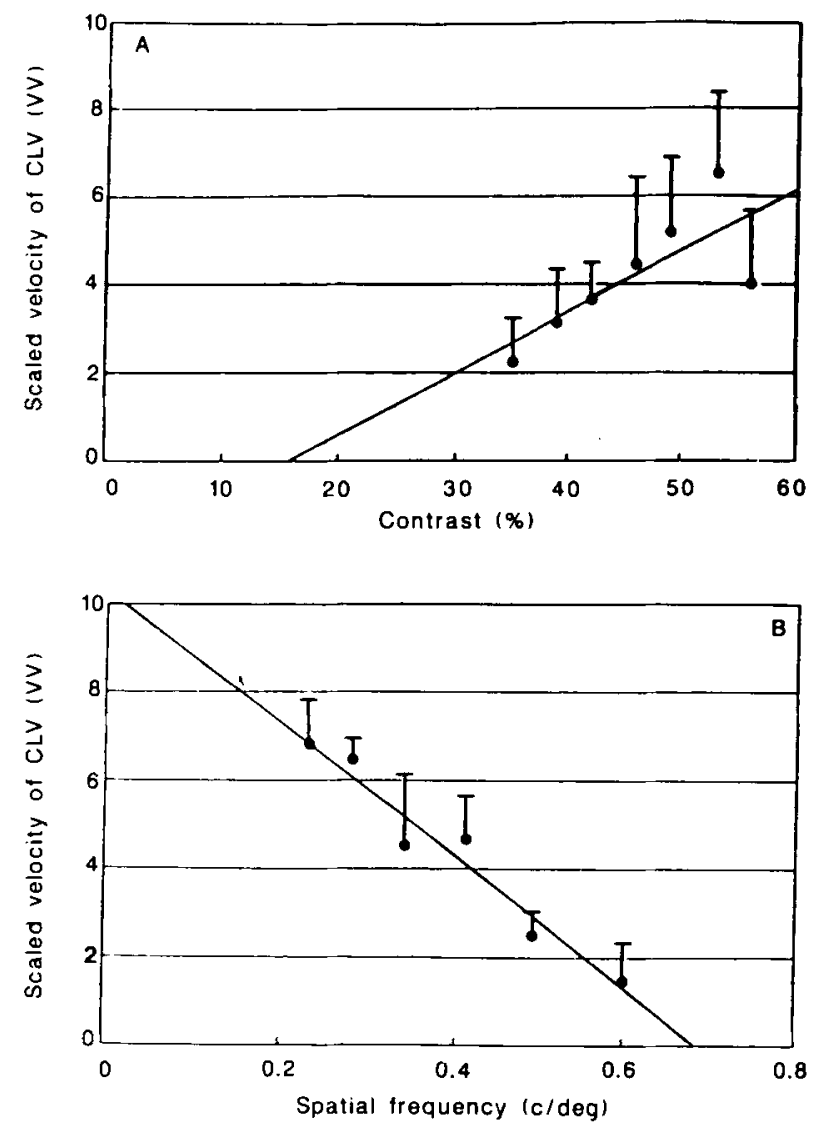

Figure 1. (A) Variation of the velocity of the perceived curvilinear vection (CLV) as a function of the contrast of the stimulation. (B) Variation of the velocity of the perceived curvilinear vection as a function of the spatial frequency of the stimulation. Average of 6 subjects ( $\bullet$ ) with corresponding sample standard deviation.

locity and perceived velocity of curvilinear vection was $V V=8.39 \cdot V-15.24$ (Figure 2B).

There was a reciprocal variation of the perceived velocity of curvilinear vection with angular velocity and contrast $[F(6,30)=5.1, p<.01]$. This result seems to indicate an interaction between velocity and contrast. There were two linear correlations: one between perceived velocity of curvilinear vection and contrast $[F(1,5)=23.79$, $p<.01 ; r=-.91]$ and another between the same perceived velocity and angular velocity $[F(1,5)=24.15$, $p<.01 ; r=.91]$. The equation of the function between angular velocity and perceived velocity of curvilinear vection was $V V=5.51 \cdot V-26.75$, and the equation between contrast and the same perceived velocity was $V V=$ $-129.06 \cdot C+102.74$. By comparing these results with those obtained in the velocity condition, it can be observed that the slopes of the equations of regression decreased only from 8.39 to 5.51 (the ratio of the slopes is equal to 1.52), whereas the slope of the equation in the condition in which contrast and velocity varied reciprocally is equal to -129.06 . These facts suggest a preeminence of the velocity of the stimulation over its contrast when the 

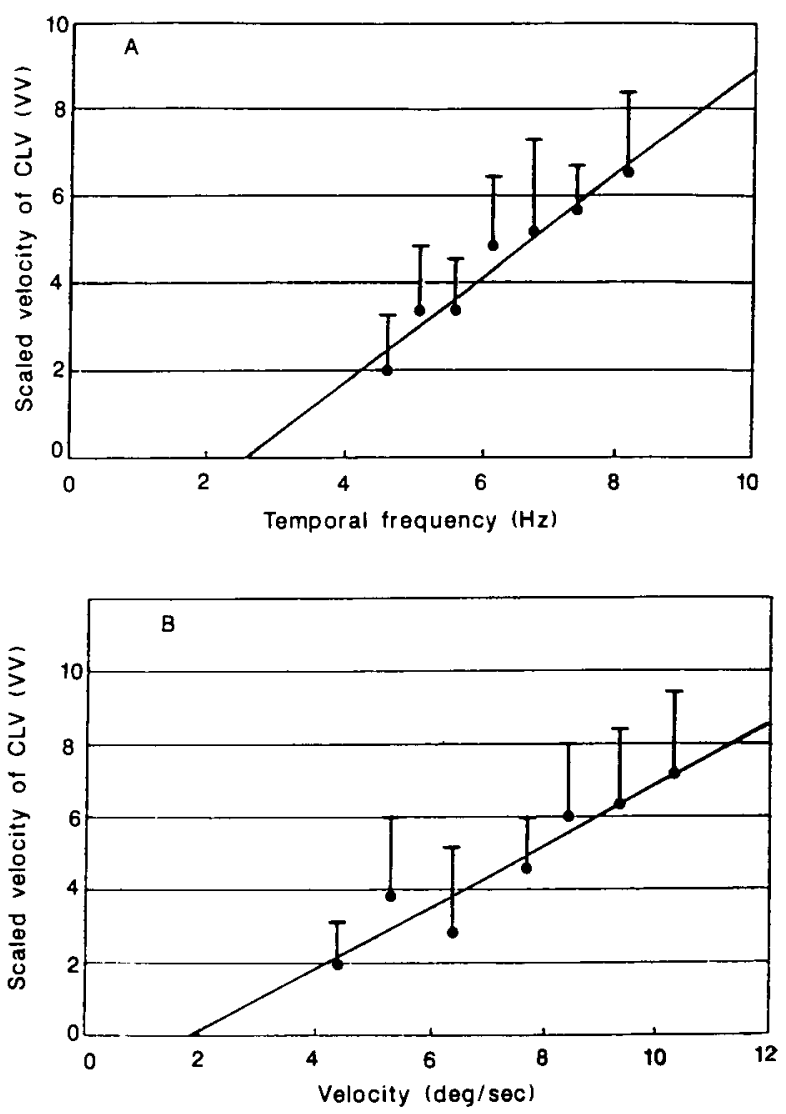

Figure 2. (A) Variation of the velocity of the perceived curvilinear vection as a function of the temporal frequency of the stimulation. (B) Variation of the velocity of the perceived curvilinear vection as a function of the angular velocity of the stimulation. Average of 6 subjects $(\bullet)$ with corresponding sample standard deviation.

perceived velocity of curvilinear vection is estimated. Contrast has only a secondary effect. It attenuated the subjects' responses with respect to perceived velocity of curvilinear vection.

\section{EXPERIMENT 2}

The purpose of this experiment was to observe the effect of the variations of contrast, spatial frequency, temporal frequency, and angular velocity (or contrast and angular velocity varying reciprocally) on the perceived degree of curvature of curvilinear vection.

\section{Method}

The conditions and procedure used in this experiment were exactly the same as those used in Experiment 1. The task for the subjects was to estimate, again using an 11-point scale, the perceived degree of curvature of curvilinear vection. The magnitude of their estimation increased with the degree of curvature (the higher the degree of curvature, the larger the curved path). Subjects M.B., N.B., R.P., E.R., C.T., and X.S. took part in Experiment 2.

\section{Results and Discussion}

As in Experiment 1, abscissa values represent stimuli for one eye. The perceived degree of curvature of curvilinear vection varied only with spatial frequency $[F(5,25)$ $=7.78, p<.01]$ or angular velocity $[F(6,30)=3.71$, $p<.01]$. These variations are shown in Figure 3.

This variation was linear for spatial frequency $[F(1,4)$ $=118.71, p<.01 ; r=-.984]$ and also for angular velocity $[F(1,4)=17.32, p<.01 ; r=.88]$. The equation of the linear function between spatial frequency and perceived degree of curvature of curvilinear vection $(D C)$ was $D C=-131.53 \cdot S F+93$ (Figure $3 \mathrm{~A}$ ) and that between angular velocity and perceived degree of curvature was $D C=6.11 \cdot V+0.29$ (Figure $3 \mathrm{~B}$ ).

The ratio between the slopes of the linear functions for perceived velocity and perceived degree of curvature of curvilinear vection with spatial frequency was 1.14 . The ratio was 1.37 when the angular velocity was the independent variable. The relationship between perceived veloc-
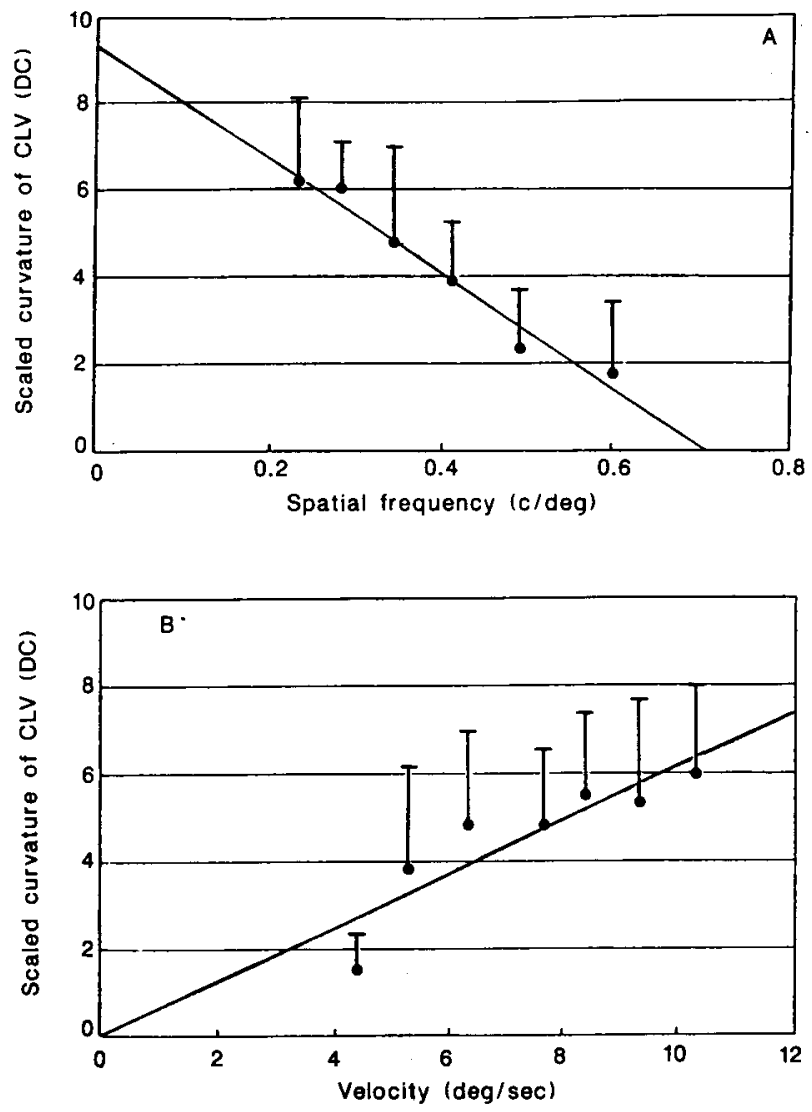

Figure 3. (A) Variation of the degree of curvature of the perceived curvilinear vection as a function of the spatial frequency of the stimulation. (B) Variation of the degree of curvature of the perceived curvilinear vection as a function of the angular velocity of the stimulation. Average of 6 subjects $(\bullet)$ with corresponding sample standard deviation. 
ity and degree of curvature of curvilinear vection has therefore been determined.

There was a linear relationship between these two attributes of curvilinear vection when the spatial frequency varied $[F(1,4)=124.87, p<.01 ; r=.984]$, the equation of this linear function being $V V=1.13 \cdot D C-2.84$. There was also a linear relationship between these variables when the velocity varied $[F(1,5)=11.26, p<.03$; $r=.832$ ], the equation of this linear function being $V V$ $=1.06 \cdot D C-1.34$.

Results obtained during Experiments 1 and 2 show that perceived velocity and degree of curvature of curvilinear vection, two attributes of that induced self-motion, varied differently with parameters that define a visual scene, such as contrast, spatial frequency, temporal frequency, and angular velocity. Indeed, the perceived velocity of curvilinear vection varied with contrast, spatial frequency, temporal frequency, and angular velocity, whereas the perceived degree of curvilinear vection varied only with spatial frequency and angular velocity. In the same way, it was observed that perceived degree of curvature did not vary with spatial frequency, whereas perceived velocity still varied with angular velocity when apparent depth was present in the visual scene. Two vertical sinewave drifting gratings displayed on the screen of each monitor having different spatial frequencies and velocities induced apparent depth. That observation confirms the different variations of the attributes of curvilinear vection as a function of the spatiotemporal parameters used in the present study.

\section{EXPERIMENT 3}

It has been shown that the latency of rectilinear vection (Berthoz et al., 1975) or circular vection (Brandt, Dichgans, \& Koenig, 1973) does not vary with the velocity of the stimulation. The aim of Experiment 3 was to study variations of the latency and time of stabilization of curvilinear vection as a function of contrast, spatial frequency, temporal frequency, and angular velocity (or contrast and angular velocity varying reciprocally).

\section{Method \\ The same conditions as those used in Experiments 1 and 2 were presented to four trained subjects (M.B., N.B., R.P., and X.S.). The system of response was a joystick connected with a Z80 micro- computer. The subjects' task was to move the joystick when they began to perceive curvilinear vection and then to stop their move- ment when vection was stabilized. The latency of curvilinear vec- tion and its time of stabilization were measured from the time 0 , that is, the beginning of each trial. The duration of each trial was $22 \mathrm{sec}$. A preliminary study showed that this duration was quite sufficient to perceive steady curvilinear vection.}

\section{Results and Discussion}

The results show that latency and time of stabilization of curvilinear vection did not vary significantly with contrast, spatial frequency, temporal frequency, angular velocity, or the combination of the latter with contrast. Consequently, it seems that there is no relationship between these variables and latency and time of stabilization of curvilinear vection. Mean latency and mean time of stabilization for the 4 subjects and the five conditions were 2.40 and $8.37 \mathrm{sec}$, respectively. Mean latencies for each subject for all conditions ranged from 1.15 to $5.67 \mathrm{sec}$; mean times of stabilization ranged from 5.64 to $11.93 \mathrm{sec}$. The mean latency that Berthoz et al. (1975) measured for rectilinear vection was between 1 and $2 \mathrm{sec}$, which is in the same range as the lowest mean latency per subject measured in the present study

\section{GENERAL DISCUSSION}

Since the subjects in this study of curvilinear vection were in a state of dichoptic vision, all observed variations of perceived curvilinear vection should have required interhemispheric processing of visual information.

It was shown in Experiment 1 that perceived velocity of curvilinear vection decreased with spatial frequency. This result is not consistent with that found for rectilinear vection (Berthoz \& Droulez, 1982), which may be related to the fact that the amplitude of postural readjustments varies logarithmically with spatial frequency (Lestienne, Soechting, \& Berthoz, 1977). But the decrease with spatial frequency is consistent, on the one hand, with the existence of a spatiotemporal zone of perception of linear vections because curvilinear vection was perceived only if the spatial frequency was below $1 \mathrm{c} / \mathrm{deg}$ and, on the other hand, with the value of the optimum of perception of linear vections for spatial frequency, which is about $0.50 \mathrm{c} / \mathrm{deg}$, because this is a low spatial frequency value (Sauvan \& Bonnet, 1988, 1989).

In the same way as in other induced self-motions in peripheral vision (Berthoz \& Droulez, 1982; Dichgans \& Brandt, 1978), it was shown in the present study that perceived velocity of curvilinear vection increases with angular velocity. This result differs from those of central rectilinear vection studies that found a decrease in induced self-motion with increased stimulation velocity (Andersen \& Braunstein, 1985). The inducing stimulus used by Andersen and Braunstein was a radially expanding depth pattern rather than the drifting sine-wave gratings used in the present study. Consequently, both the optical flow position in the observer's visual field and the optical flow structure would play a role in judging the perceived velocity of vection, with the optical flow defined as the changing structure of light at an eye caused by the movement of an organism relative to its environment (see Gibson, 1979). Correspondingly, it has been shown that different classes of optical flow correspond to different classes of self-motions such as translational and circular movement (Warren, Mestre, Blackwell, \& Morris, 1991).

Observed variations of the perceived velocity of curvilinear vection as a function of the stimulus angular velocity and spatial frequency show that the experienced velocity of curvilinear vection is dependent on the temporal and spatial characteristics of the stimulation, in the same manner as is circular vection (de Graaf, Wertheim, Bles, \& Kremers, 1990). In the present experimental 
work, the subjects were suddenly exposed to a moving stimulation at the beginning of each trial. This corresponded to a very high visual acceleration. It has been shown that latency of circular vection has a minimum at $5 \mathrm{deg} / \mathrm{sec}^{2}$ and increases for slower and faster accelerations of the visual stimulus (Melcher \& Henn, 1981). The latency of curvilinear vection, a linear vection with a circular component, could also vary as a function of the visual stimulation acceleration, although this was not investigated in Experiment 3.

Our results show that attributes of curvilinear vection vary differently with contrast, spatial frequency, temporal frequency, and angular velocity. Especially, the perceived velocity of curvilinear vection varies with contrast, spatial frequency, temporal frequency, and angular velocity, and the perceived degree of curvature of curvilinear vection varies only with spatial frequency and angular velocity. Consequently, unlike the perceived velocity, the perceived degree of curvature is not always unambiguously estimated, so that the perceived degree of curvature and the perceived velocity are not always adjusted. That discrepancy and the fact that the perceived direction of curvilinear vection is not always unambiguously perceived (Sauvan \& Bonnet, 1989) indicate that the peripheral visual information is not sufficient to compute the curvilinear component of induced self-motion in a curved path. The central part of the visual field plays a role in perceiving complex self-motion such as circular movement (Mestre \& Warren, 1989; Warren et al., 1991). Accordingly, two systems appear to control ambient vision: a lower level system that would require peripheral vision and a higher level system that would work in the central visual field and would use complex visual information such as depth cues (Andersen \& Braunstein, 1985). The primary characteristic of the more primitive system seems to be its sensitivity to low spatial frequencies. Indeed, spatial frequencies below $1 \mathrm{c} / \mathrm{deg}$ define the spatiotemporal zone of perception of peripheral linear vections so that the optimum of perception of peripheral linear vections for spatial frequency is about $0.50 \mathrm{c} / \mathrm{deg}$ (Sauvan \& Bonnet, 1988, 1989). Moreover, the more primitive ambient system has been described as complementary to the focal system-the former system underlying spatial orientation and localization, and the latter system underlying object recognition (Ingle \& Sprague, 1975). Correspondingly, the more primitive system may be related to the parietal visual processing stream, and the focal system may be related to the temporal visual processing stream (a recent description of these pathways can be found in Boussaoud, Ungerleider, \& Desimone, 1990). The higher level ambient system of Andersen and Braunstein (1985) should be related to sites of interaction between these two visual processing streams. Several sites of integration of visual inputs relayed over the parietal and temporal visual pathways have been identified (Morel \& Bullier, 1990). Some observations indicate that it is important to take into account not only the visual inputs and processing properties of these systems but also the pat- tern of their output connections (Goodale, Milner, Jakobson, \& Carey, 1991). In short, to understand the visual control of daily actions such as locomotion or driving a vehicle, it would be interesting to study variations of complex induced self-motions as a function of central and/or peripheral visual stimulations. Indeed, this study would not only allow investigators to compare higher and lower level ambient systems but also would allow them to determine the effects on complex induced self-motions of various combinations of stimulations that are more effective in central or peripheral vision.

\section{REFERENCES}

Andersen, G. J. (1986). Perception of self-motion: Psychophysical and computational approaches. Psychological Bulletin, 99, 52-65.

Andersen, G. J., \& Braunstein, M. L. (1985). Induced self-motion in central vision. Joumal of Experimental Psychology: Human Perception \& Performance, 11, 122-132.

Berthoz, A., \& Droulez, J. (1982). Linear self-motion perception. In A. H. Wertheim, W. A. Wagenaar, and H. W. Leibowitz (Eds.), Tutorials in motion perception (pp. 157-199). London: Plenum.

Berthoz, A., Pavard, B., \& Young, L. R. (1975). Perception of linear horizontal self-motion induced by peripheral vision (linear vection): Basic characteristics and visual-vestibular interactions. Experimental Brain Research, 23, 471-489.

Bonnet, C. (1986). Manuel pratique de psychophysique. Paris: Armand Colin.

Boussaoud, D., Ungerleider, L. G., \& Desimone, R. (1990). Pathways for motion analysis: Cortical connections of the medial superior temporal and fundus of the superior temporal visual areas in the macaque. Journal of Comparative Neurology, 296, 462-495.

Brandt, Th., Dichgans, J., \& Koenig, E. (1973). Differential effects of central versus peripheral vision on egocentric and exocentric motion perception. Experimental Brain Research, 16, 476-491.

BütTner, U., \& Buettner, U. V. (1978). Parietal cortex (2v) neuronal activity in the alert monkey during vestibular and optokinetic stimulation. Brain Research, 153, 392-397.

BütTNer, U., \& HeNN, V. (1976). Thalamic unit activity in the alert monkey during natural vestibular stimulation. Brain Research, 103, 127-132.

Büttner, U., \& HenN, V. (1981). Circularvection: Psychophysics and single-unit recordings in the monkey. Annals of the New York Academy of Sciences, 374, 274-283.

BütTner, U., \& LANG, W. (1979). The vestibulocortical pathway: Neurophysiological and anatomical studies in the monkey. Progress in Brain Research, 50, 581-588.

de Graaf, B., Wertheim, A. H., Bles, W., \& Kremers, J. (1990). Angular velocity, not temporal frequency determines circular vection. Vision Research, 30, 637-646.

Dichgans, J., \& BRANDT, TH. (1978). Visual-vestibular interaction: Effects on self-motion perception and postural control. In R. Held, H. W. Leibowitz, \& H. L. Teuber (Eds.), Handbook of sensory physiology (Vol. 8, pp. 755-804). Berlin: Springer-Verlag.

GiBson, J. J. (1979). The ecological approach to perception. Boston: Houghton Mifflin.

Goodale, M. A., Milner, A. D., Jakobson, L. S., \& Carey, D. P. (1991). A neurological dissociation between perceiving objects and grasping them. Nature, 349, 154-156.

How ARD, I. P. (1986). The perception of posture, self motion and visual vertical. In K. R. Boff, L. Kaufman, \& J. P. Thomas (Eds.), Handbook of perception and human performance (Vol. 1, pp. 18-1-18-62). New York: Wiley.

Hulk, J., \& REMPT, F. (1983). Vertical optokinetic sensations by limited stimulation of the peripheral field of vision. Ophthalmologica, 186, 97-103.

InGLe, D., \& SpRague, J. M. (1975). Sensorimotor function of the midbrain tectum. Neurosciences Research Program Bulletin, 13, 244-251. 
Leibowitz, H. W., Rodemer, C. S., \& Dichgans, J. (1979). The independence of dynamic spatial orientation from luminance and refractive error. Perception \& Psychophysics, 25, 75-79.

Lestienne, F., Soechting, J., \& Berthoz, A. (1977). Postural readjustments induced by linear motion of visual scenes. Experimental Brain Research, 28, 363-384.

Longuet-Higgins, H. C., \& Prazdny, K. (1980). The interpretation of a moving retinal image. Proceedings of the Royal Society of London, Series $B, 208,387-397$.

MelCher, G. A., HenN, V. (1981). The latency of circular vection during different accelerations of the optokinetic stimulus. Perception \& Psychophysics, 30, 552-556.

Mestre, D., \& WARReN, W. H. (1989). Le flux optique: Son rôle lors du contrôle du déplacement. Psychologie Françise, 34, 5-11.

Morel, A., \& Bullier, J. (1990). Anatomical segregation of two cortical visual pathways in the macaque monkey. Visual Neuroscience, 4. 555-578.

Orban, G. A. (1984). Neuronal operations in the visual cortex. Berlin: Springer-Verlag.

Post, R. B. (1988). Circular vection is independent of stimulus eccentricity. Perception, 17, 737-744.

Prazdny, K. (1981). Determining the instantaneous direction of motion from optical flow generated by a curvilinear moving observer. Computer Graphics \& Image Processing, 17, 238-248.

Prazdny, K. (1983). On the information in optical flows. Computer Vision, Graphics, \& Image Processing, 22, 239-259.

Probst, Th., Straube, A., \& Bles, W. (1985). Differential effects of ambivalent visual-vestibular-somatosensory stimulation on the perception of self-motion. Behavioural Brain Research, 16, 71-79.

RIEGER, J. H. (1983). Information in optical flows induced by curved paths of observation. Joumal of the Optical Sociery of America, 73 339-344.

Sauvan, X. M., Bonnet, C. (1988, March). Thresholds and variations of the forward rectilinear and curvilinear vections in man. In Visual processing of form and motion. Proceedings of the EBBS workshop, Tübingen, Germany.

Sauvan, X. M., Bonnet, C. (1989). Les sensations de déplacement curvilinéaire générées visuellement. Psychologie Française, 34, 19-24.

WAESPE, W., HENN, V. (1977). Neuronal activity in the vestibular nuclei of the alert monkey during vestibular and optokinetic stimulation. Experimental Brain Research, 27, 533-538

Waespe, W., \& HenN, V. (1978). Conflicting visual-vestibular stimulation and vestibular nucleus activity in alert monkeys. Experimental Brain Research, 33, 203-211.

Warren, W. H., Mestre, D. R., Blackwell, A. W., Morris, M. W. (1991). Perception of circular heading from optical flow. Journal of Experimental Psychology: Human Perception \& Performance, 17, 28-43.

WURTZ, R. H., \& DuFfY, C. J. (1992). Neuronal correlates of optic flow stimulation. Annals of the New York Academy of Sciences, 656, 205-219.

(Manuscript received December 14, 1987; revision accepted for publication October 6, 1992.)

Forthcoming Articles (continued from page 428)

eralization to a novel displacement" by R.B. Welch, B. Bridgeman, S. Anand, \& K.E. Browman (R.B.W., NASA-Ames Research Ctr., Mail Stop 239-11, Moffett Field, CA 94035)

"Stereo-motion cooperation and the use of motion disparity in the visual perception of 3D structure" by V. CornilleauPeres \& J. Droulez (V.C., Lab. de Physiologie Neurosensorielle, 15 rue de l'Ecole de Medecine, 75270 Paris cedex 06, France)

"Organizational factors and the perception of motion in depth" by D.H. Mershon, T.A. Jones, \& M.E. Taylor (D.H.M., Dept. of Psychology, North Carolina State Univ., Box 7801, Raleigh, NC 27695-7801)

"Visual angle as a determinant of perceived inter-object distance' 'by C.A. Levin \& R.N. Haber (C.A.L., 3268 Summit Ave., Highland Park, IL 60035)

"Temporal constraints on apparent motion in auditory space" by S. Lakatos (Dept. of Psychology, Jordan Hall, Bldg. 420, Stanford Univ., Stanford, CA 94305)

"Limits on the limitations of context conditioned effects in the perception of $[b]$ and [w]' by J.L. Miller \& S.C. Wayland (J.L.M., Dept. of Psychology, Northeastern Univ., Boston, MA 02115)

"Viewing behavior: Ocular and attentional disengagement" by W.J. Wa \& L.B. Stelmach (W.J.W., Communications Research Ctr., 3701 Carling Ave., Ottawa, ONT K2H 8S2, Canada)

"Detection of three-dimensional surfaces from optic flow: The effects of noise" by G.J. Andersen \& A.P. Wuestefeld (G.J.A., Dept. of Psychology, Univ. of California, Riverside, CA 92521-0426)

"Estimating local shape from shading in the presence of global shading' by R.G.F. Erens, A.M.L. Kappers, \& J.J. Koenderink (R.G.F.E., Univ. of Utrecht, Princetonplein 5, 3584 CC Utrecht, Holland)

“What are human express saccades?'” by A. Kingstone \& R.M.
Klein (A.K., Ctr. for Neuroscience, 1633 DaVinci Ct., Univ. of California, Davis, Davis, CA 95616)

"Binocular rivalry of equiluminent targets" by D.H. Westendorf \& M.P. Galupo (D.H.W., Dept. of Psychology Univ. of Arkansas Fayetteville, AR 72701-1201)

"Both perceptual and conceptual factors influence taste-odor and taste-taste interactions"' by R.A. Frank, N.J. Van der Klaauw, \& H.N.J. Schifferstein (R.A.F., ML \#376, Dept. of Psychology, Univ. of Cincinnati, Cininnati, OH 45221-0376)

"Prismatic displacement of vision induces transient changes in the timing of eye-hand coordination" by Y. Rossetti, K. Koga, \& T. Mano (Y.R., Vision et Motricite, INSERM Univ., 94-16, Ave. du doyen Lepine, 69500 Bron, France)

"The effect of tempo and tone duration on rhythm discrimination' by S. Handel (Dept. of Psychology, Univ. of Tennessee, Knoxville, TN 37996-0900)

"A comparison of thresholds for 1/3-octave filtered clicks and noise bursts in infants and adults"' by K.M. Berg (Inst. for Adv. Study of Comm. Processes, 63 Dauer Hall, Univ. of Florida, Gainesville, FL 32611)

"Tempo sensitivity in auditory sequences: Evidence for a multiple-look model"' by C. Drake \& M.-C. Botte (C.D., Lab. de Psy. Exp., 28 rue Serpente, 75006 Paris, France)

"Duration discrimination of empty and filled intervals marked by auditory and visual signals" by S. Grondin (Dept. of Psychology, Laurentian Univ., Ramsey Lake Rd., Sudbury, Ontario, Canada P3E 2C6)

"Visual influences on auditory pluck and bow judgments" by H.M. Saldana \& L.D. Rosenblum (L.D.R., Dept. of Psychology, Univ. of Calif., Riverside, Riverside, CA 92521)

"Phonatactic knowledge of word boundaries and its use in infant speech perception"' by A.D. Friederici \& J.M.I. Wessels (A.D.F., Cognitive Science Lab. Berlin, Inst. für Psy., Freie Univ. Berlin, Habelschwerdter Alle 45, D-1000 Berlin 33, Germany) 